\title{
Comment on "A dynamic nonlinear subgrid-scale stress model" [Phys. Fluids 17, 035109 (2005)]
}

\author{
Stefan Heinz ${ }^{a)}$ \\ Department of Mathematics, University of Wyoming, 1000 East University Avenue, \\ Laramie, Wyoming 82071
}

(Received 27 February 2005; accepted 4 May 2005; published online 16 September 2005)

[DOI: $10.1063 / 1.2033107]$

In a recent paper Wang and Bergstrom introduce a dynamic nonlinear model for the deviatoric part $\tau_{i j}^{*}$ of the subgrid-scale (SGS) stress. ${ }^{1}$ In terms of their notation this model may be written as

$$
\tau_{i j}^{*}=\tau_{i j}^{*(1)}+\frac{2 C_{W}}{C_{S}|\bar{S}|} P_{i j}^{(1)}+D_{i j}^{(1)} .
$$

The first-order approximations of the SGS stress tensor $\tau_{i j}^{*(1)}$, production $P_{i j}^{(1)}$ of stress by shear, and production source term $D_{i j}^{(1)}$ are given by the expressions

$$
\begin{aligned}
& \tau_{i j}^{*(1)}=-2 \nu_{\mathrm{SGS}} \bar{S}_{i j}, \\
& P_{i j}^{(1)}=-\bar{u}_{i, k} \tau_{k j}^{*(1)}-\bar{u}_{j, k} \tau_{k i}^{*(1)}+\frac{2}{3} \bar{S}_{m n} \tau_{n m}^{*(1)} \delta_{i j}, \\
& D_{i j}^{(1)}=\frac{C_{N}+2 C_{W}}{C_{S}|\bar{S}|}\left(\bar{S}_{i k} \tau_{k j}^{*(1)}+\bar{S}_{j k} \tau_{k i}^{*(1)}-\frac{2}{3} \bar{S}_{m n} \tau_{n m}^{*(1)} \delta_{i j}\right) .
\end{aligned}
$$

In these expressions, $\bar{S}_{i j}=\left(\bar{u}_{i, j}+\bar{u}_{j, i}\right) / 2$ is the filtered rate-ofstrain tensor, and $|\bar{S}|=\left(2 \bar{S}_{i k} \bar{S}_{k i}\right)^{1 / 2}$ is a characteristic strain rate. The SGS viscosity $\nu_{\mathrm{SGS}}=C_{S} \bar{\Delta}^{2}|\bar{S}|$, where $\bar{\Delta}$ refers to the length scale associated with the filter size. To simplify the comparisons presented below the last two terms of (1) are written here in terms of $C_{S}$ and $|\bar{S}|$, but the use of $\nu_{\mathrm{SGS}}$ $=C_{S} \bar{\Delta}^{2}|\bar{S}|$ in (1) and (2a)-(2c) reveals that these two terms are actually independent of $C_{S}$ and $|\bar{S}|$. By adopting a methodology suggested by Wong, ${ }^{2}$ the model parameters $C_{S}, C_{W}$, and $C_{N}$ are calculated such that the local error function is minimized. The latter constraint implies the following linear equation system for $C_{S}, C_{W}$, and $C_{N}$ (see the corresponding definitions of Wang and Bergstrom): ${ }^{1}$

$$
\left[\begin{array}{lll}
M_{i j} M_{i j} & M_{i j} W_{i j} & M_{i j} N_{i j} \\
W_{i j} M_{i j} & W_{i j} W_{i j} & W_{i j} N_{i j} \\
N_{i j} M_{i j} & N_{i j} W_{i j} & N_{i j} N_{i j}
\end{array}\right]\left[\begin{array}{c}
C_{S} \\
C_{W} \\
C_{N}
\end{array}\right]=-\left[\begin{array}{c}
\mathcal{L}_{i j}^{*} M_{i j} \\
\mathcal{L}_{i j}^{*} W_{i j} \\
\mathcal{L}_{i j}^{*} N_{i j}
\end{array}\right] .
$$

Solutions for the model parameters $C_{S}, C_{W}$, and $C_{N}$ exist if and only if the three differential tensorial functions $M_{i j}, W_{i j}$, and $N_{i j}$ are linearly independent. The authors suspect that these three different tensorial functions are, in fact, indepen-

\footnotetext{
a)Telephone: (307) 766-4203. Fax: (307) 766-6838. Electronic mail: heinz@uwyo.edu
}

dent. However, a strict analytical proof is not yet available to support such a conjecture, and it remains an open question for further analytical explorations. ${ }^{1}$ Regarding the suitability of their dynamic nonlinear model (DNM) (19) [which is relation (1) here] as SGS stress tensor model the authors conclude lastly, the proposed DNM formula (19) is, at most, an assumption for modeling the SGS stress. Whether the rightand left-hand sides of formula (19) are satisfactorily correlated can be examined via possible future a priori approaches based on DNS and experimental data analysis. ${ }^{1}$

The latter question will be addressed here. One may distinguish two approaches to construct SGS stress tensor models. One way (the approach of Wang and Bergstrom) is to parametrize this tensor directly by adopting heuristic assumptions. Models obtained in this way are not shown to be correct; one needs direct numerical simulation (DNS) or experimental data to demonstrate the suitability of the assumptions applied. The conclusions obtained by such comparisons then depend on the specific flow configuration considered, and the Reynolds number and grid spacing applied. In addition to that, the way to construct such models does not prove the general existence of solutions to such model equations. An alternative to the construction of heuristic SGS stress tensor models is to explain the underlying physics. The SGS stress tensor is the result of the correlations of SGS velocity fluctuations. One needs, therefore, a physically correct model for dynamics of SGS velocity fluctuations in order to derive then the SGS stress tensor as a consequence. The latter approach has some obvious advantages compared to the application of heuristic SGS stress tensor models. First, the general existence of solutions to model equations for SGS velocity fluctuations proves the general existence of solutions to filtered velocity equations. Second, there are first principles available for the construction of physically consistent models for SGS velocity fluctuations, ${ }^{3}$ which provides support for the proof of the suitability of such models. Third, the consistency between underlying equations for SGS fluctuations and SGS stress tensor models (this means the existence of a systematic hierarchy of simple and more complex models) is helpful for the understanding of the range of applicability of algebraic SGS stress tensor models. It provides alternative methods (transport equations for the stress tensor and velocity fluctuations) which may be applied under conditions where the use of algebraic SGS stress tensor models appears to be questionable, and it enables the development of consistent hybrid methods for velocity and reacting scalar 
fields. ${ }^{3}$ SGS velocity models that are required to apply this approach were developed recently. They were shown to have a consistent theoretical basis, and their predictions agree well with the DNS data and experimental results. ${ }^{3-7}$ By following a recent publication of the author, ${ }^{7}$ their implications for the SGS stress tensor will be shown here in order to address the suitability of Wang and Bergstrom's SGS stress model (1)-(3).

By adopting the simplified Langevin model for SGS velocity dynamics, one may derive the following equation for the deviatoric part of the SGS stress tensor in incompressible flows: ${ }^{7}$

$$
\frac{\partial \tau_{i j}^{*}}{\partial t}+\bar{u}_{k} \frac{\partial \tau_{i j}^{*}}{\partial x_{k}}+\frac{\partial T_{i j k}}{\partial x_{k}}=P_{i j}-4 \frac{\nu_{r}}{\tau_{L}} \bar{S}_{i j}-\frac{2}{\tau_{L}} \tau_{i j}^{*} .
$$

Here, $\mathrm{T}_{i j k}$ represents contributions due to triple correlations, and

$$
P_{i j}=-\bar{u}_{i, k} \tau_{k j}^{*}-\bar{u}_{j, k} \tau_{k i}^{*}+\frac{2}{3} \bar{S}_{m n} \tau_{n m}^{*} \delta_{i j}
$$

represents the production of stress by shear. The residual viscosity is given by $\nu_{r}=k_{r} \tau_{L} / 3$, where $k_{r}$ denotes the residual turbulent kinetic energy and $\tau_{L}$ refers to the characteristic time scale of velocity fluctuations. By assuming a local equilibrium between production and dissipation [by neglecting the left-hand side of (4)] we obtain

$$
\tau_{i j}^{*}=-2 \nu_{r} \bar{S}_{i j}+\frac{\tau_{L}}{2} P_{i j}
$$

In the second order of approximation, relation (6) implies the nonlinear SGS stress tensor model derived by the author, ${ }^{7}$

$$
\tau_{i j}^{*}=\tau_{i j}^{*(1)}+\frac{2 C_{W}}{C_{S}|\bar{S}|} P_{i j}^{(1)} .
$$

Here, $\tau_{i j}^{*(1)}$ and $P_{i j}^{(1)}$ are given by (2a) and (2b); this means $\nu_{\mathrm{SGS}}=\nu_{r}$. To simplify the model comparison below, $\tau_{L}$ is taken in (7) with reference to $|\bar{S}|$ : we assumed that $\tau_{\mathrm{L}}$ $=4 C_{W} /\left(C_{S}|\bar{S}|\right)$. The model parameters in (7) can be obtained by adopting a dynamic procedure. ${ }^{7}$ In terms of the notation used by Wang and Bergstrom the corresponding equations are given by

$$
\left[\begin{array}{cc}
M_{i j} M_{i j} & M_{i j} A_{i j} \\
A_{i j} M_{i j} & A_{i j} A_{i j}
\end{array}\right]\left[\begin{array}{c}
C_{S} \\
C_{W}
\end{array}\right]=-\left[\begin{array}{c}
\mathcal{L}_{i j}^{*} M_{i j} \\
\mathcal{L}_{i j}^{*} A_{i j}
\end{array}\right],
$$

where $A_{i j}=W_{i j}-2 N_{i j}$ is introduced. The solutions of (8) always exist since $M_{i j}$ and $A_{i j}$ are linearly independent: $A_{i j}$ involves the filtered rate-of-rotation tensor $\bar{\Omega}_{i j}$ whereas $M_{i j}$ is independent of $\bar{\Omega}_{i j}$.

The models (1)-(3) of Wang and Bergstrom and (7) and (8) of Heinz differ by the appearance of $D_{i j}^{(1)}$ in (1) [which is nonzero in general: see (3)]. What are the advantages and disadvantages of considering a nonzero $D_{i j}^{(1)}$ ? One may assume that it is an advantage to have the additional adjustable parameter $C_{N}$ (a more flexible method) available. However, one has to pay for that a price due to the fact that physically consistent dynamics of velocity fluctuations with correlations as specified by (1) [this means with a nonzero $D_{i j}^{(1)}$ ] do not exist [see the derivation of (7)]. First, the general existence of solutions to corresponding filtered velocity equations cannot be shown by demonstrating the general existence of underlying velocity fluctuations: it is then at least unclear whether such solutions do exist in general. Second, realizability (the constraint that any moment and correlation transport equations considered should represent realizable equations for moments and correlations of a stochastic process) was proved to represent a valuable guiding principle for turbulence modeling. ${ }^{8-11}$ Correspondingly, the use of realizable models was found to be of remarkable relevance regarding the application of probability density function (PDF) and Reynolds-averaged Navier-Stokes (RANS) methods. ${ }^{12-14} \mathrm{~A}$ corresponding importance of realizable models has to be expected with regard to the accuracy of models for dynamics of SGS variables. Third, inconsistencies of SGS stress tensor models and models for SGS fluctuations are not helpful for the use of SGS stress tensor models as part of more general methods (hybrid methods for velocity and reacting scalar fields).

The three disadvantages of the model (1)-(3) described above can be avoided by applying the model (7) and (8). The use of (7) and (8) represents, therefore, at least a valid alternative to the use of (1)-(3). Obviously, a more complete insight into the differences between these two models requires extensive comparisons of their performance with regard to simulations of various flows.

${ }^{1}$ B. C. Wang and D. J. Bergstrom, "A dynamic nonlinear subgrid-scale stress model," Phys. Fluids 17, 035109 (2005).

${ }^{2}$ V. C. Wong, "A proposed statistical-dynamic closure method for the linear or nonlinear subgrid-scale stresses," Phys. Fluids A 4, 1080 (1992).

${ }^{3}$ S. Heinz, Statistical Mechanics of Turbulent Flows (Springer, Berlin, 2003).

${ }^{4}$ F. A. Jaberi, P. J. Colucci, S. James, P. Givi, and S. B. Pope, "Filtered mass density function for large-eddy simulation of turbulent reacting flows," J. Fluid Mech. 401, 85 (1999).

${ }^{5}$ L. Y. M. Gicquel, P. Givi, F. A. Jaberi, and S. B. Pope, "Velocity filtered density function for large eddy simulation of turbulent flows," Phys. Fluids 14, 1196 (2002).

${ }^{6}$ M. R. H. Sheikhi, T. G. Drozda, P. Givi, and S. B. Pope, "Velocity-scalar filtered density function for large-eddy simulation of turbulent flows," Phys. Fluids 15, 2321 (2003).

${ }^{7}$ S. Heinz, "On Fokker-Planck equations for turbulent reacting flows. Part 2. Filter density function for large eddy simulation," Flow, Turbul. Combust. 70, 153 (2003).

${ }^{8} \mathrm{U}$. Schumann, "Realizability of Reynolds stress turbulence models," Phys. Fluids 20, 721 (1977).

${ }^{9}$ J. L. Lumley, "Computational modeling of turbulent flows," Adv. Appl. Mech. 18, 123 (1978).

${ }^{10}$ P. A. Durbin and C. G. Speziale, "Realizability of second-moment closure via stochastic analysis," J. Fluid Mech. 280, 395 (1994).

${ }^{11} \mathrm{~S}$. B. Pope, "On the relationship between stochastic Lagrangian models of turbulence and second-moment closures," Phys. Fluids 6, 973 (1994).

${ }^{12}$ M. Muradoglu, P. Jenny, S. B. Pope, and D. A. Caughey, "A consistent hybrid finite-volume/particle method for the PDF equations of turbulent reactive flows," J. Comput. Phys. 154, 342 (1999).

${ }^{13}$ P. A. Nooren, H. A. Wouters, T. W. J. Peeters, and D. Roekaerts, "Monte Carlo PDF modeling of a turbulent natural-gas diffusion flame," Combust. Theory Modell. 1, 79 (1997).

${ }^{14}$ H. A. Wouters, P. A. Nooren, T. W. J. Peeters, and D. Roekaerts, "Simulation of a bluff-body stabilized diffusion flame using second-moment closure and Monte Carlo methods," in The 26th Symposium (International) on Combustion (The Combustion Institute, Pittsburgh, 1996), pp. 177-185. 